\title{
Application of large-area avalanche photodiodes to X-ray spectrometry of muonic atoms
}

L.M.P. Fernandes ${ }^{\mathrm{a}}$, A. Antognini ${ }^{\mathrm{b}}$, M. Boucher ${ }^{\mathrm{c}}$, C.A.N. Conde ${ }^{\mathrm{a}}$, O. Huot ${ }^{\mathrm{c}}$, P.E. Knowles ${ }^{\mathrm{c}}$, F. Kottmann ${ }^{\text {d }}$, L. Ludhova ${ }^{\mathrm{c}}$, F. Mulhauser ${ }^{\mathrm{c}, *}$, R. Pohl ${ }^{\mathrm{b}, \mathrm{e}}$, J.M.F. dos Santos ${ }^{\mathrm{a}}$, L.A. Schaller ${ }^{\mathrm{c}}$, D. Taqqu ${ }^{\mathrm{e}}$, J.F.C.A. Veloso ${ }^{\mathrm{a}}$

a Departamento de Física da Universidade de Coimbra, 3004-516 Coimbra, Portugal

${ }^{\mathrm{b}}$ Max-Planck-Institut für Quantenoptik, DE-85748 Garching, Germany

'Département de Physique de l'Université, CH-1700 Fribourg, Switzerland

${ }^{\mathrm{d}}$ Institut für Teilchenphysik, ETHZ, CH-8093 Zurich, Switzerland

e Paul Scherrer Institut, CH-5232 Villigen-PSI, Switzerland

Received 10 November 2002; accepted 16 May 2003

\begin{abstract}
Large-area avalanche photodiodes have been investigated as $1.9-\mathrm{keV} \mathrm{X}$-ray detectors for the muonic hydrogen Lamb-shift experiment. We report experimental tests carried out for evaluation of the avalanche photodiode capabilities for X-ray detection in the intense radiation and low counting rate environment of experiments with muonic atoms. Several muonic atoms were used and it was shown that the electronic background of muonic atom X-ray spectra can be reduced simply by timing the X-ray signal against the gate signal produced by the muon entrance. Furthermore, the background can be eliminated using coincidences between the X-ray signal and the signal resulting from the electron due to the muon decay. This coincidence discrimination results, however, in a reduction of the X-ray detection efficiency.
\end{abstract}

(c) 2003 Elsevier B.V. All rights reserved.

Keywords: Lamb shift; Muonic atoms X-ray spectrometry; Avalanche photodiodes

\section{Introduction}

An experiment to measure the energy difference between atomic states $2 \mathrm{~S}-2 \mathrm{P}$ (Lamb shift) in

\footnotetext{
is This paper was presented at the 9th Symposium on Total Reflection X-Ray Fluorescence Analysis and Related Methods, held in Madeira, Portugal, September 2002, and is published in the Special Issue of Spectrochimica Acta Part B, dedicated to that conference.

*Corresponding author. Tel.: +41-26-3009060; fax: +4126-3009747.

E-mail address: francoise.mulhauser@unifr.ch (F. Mulhauser).
}

muonic hydrogen $(\mu p)$ is undergoing at the Paul Scherrer Institute (PSI) [1,2]. The experiment consists of obtaining long-lived $\mu \mathrm{p}$ atoms in the $2 \mathrm{~S}$ metastable state by stopping low energy negative muons $\left(\mu^{-}\right)$in a small volume of lowpressure hydrogen in a $5 \mathrm{~T}$ magnetic field. A pulsed beam from a tunable laser $(\sim 6 \mu \mathrm{m}$ wavelength) induces the $2 \mathrm{~S}-2 \mathrm{P}$ transition in $\mu \mathrm{p}$. Muonic atoms in the $2 \mathrm{P}$ state decay immediately to the ground state and 1.9-keV X-rays are emitted (Fig. 1). Since the atomic energy levels of muonic hydrogen are a factor of 186 more energetic than 


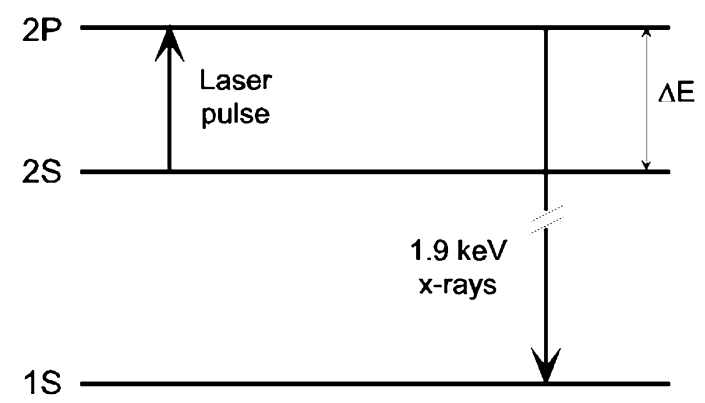

Fig. 1. Muonic hydrogen atomic levels, showing the transitions taking place in the Lamb-shift $(\Delta E)$ experiment.

those of normal hydrogen, according to the ratio of reduced masses, the transitions in $\mu p$ lie in the soft $\mathrm{X}$-ray region.

The $2 \mathrm{~S}-2 \mathrm{P}$ energy difference $(\Delta E)$ can be determined by measuring, as function of the laser wavelength, the number of coincidences between the laser pulse and the delayed 1.9-keV photon. The contribution of the RMS proton charge radius to the Lamb shift in $\mu p$ amounts to $2 \%$. Measuring the Lamb shift to a precision of $30 \mathrm{ppm}$ will allow us to deduce the RMS proton charge radius with $0.1 \%$ accuracy, 20 times more precise than presently known $[3,4]$.

The X-ray detector should be compact and insensitive to a $5 \mathrm{~T}$ magnetic field. To achieve the attended accuracy, we need an energy resolution of approximately $20 \%$ for $1.9-\mathrm{keV}$ X-rays. To differentiate between prompt $\mathrm{X}$-rays resulting from the $2 \mathrm{P}-1 \mathrm{~S}$ transition of muonic cascade in hydrogen and laser-induced delayed events, a time resolution better than 100 ns is also necessary. Large-area avalanche photodiodes (LAAPDs) are compact, monolithic devices suitable for soft Xray spectrometry [5] and can operate in magnetic fields up to $5 \mathrm{~T}$ with negligible performance degradation [6,7]. They also match the other experimental requirements: they present good time resolution and the energy resolution can be reduced to the desired values because its performance in $\mathrm{X}$-ray detection can be improved by decreasing the operating temperature [8]. Therefore, LAAPDs of $16 \mathrm{~mm}$ diameter [9] will be used during the first run, in September/October 2002. Because low counting rates are expected, the LAAPD limited area $\left(200 \mathrm{~mm}^{2}\right)$ is a drawback. However, a series of 12 LAAPDs will be used in the final experiment.

We report experimental tests carried out for evaluation of the capabilities of LAAPDs in the detection of muonic atom $(\mu p, \mu \mathrm{He}, \mu \mathrm{C}) \mathrm{X}$-rays in the intense radiation environment of the experiment. The main aim was to verify the ability of detecting the 1.9-keV X-rays, corresponding to the $2 \mathrm{P}-1 \mathrm{~S}$ transition of $\mu \mathrm{p}$, and to study the possibilities of reducing the huge X-ray spectra background present on such muonic atom experiments.

\section{Experimental setup}

The realization of the $\mu p$ Lamb-shift experiment requires a very low energy muon beam line. However, the reported tests were obtained from a different muon beam line, the $\mu \mathrm{E} 4$ area at PSI. This beam line was designed to produce muons in the $30-100 \mathrm{MeV} / \mathrm{c}$ momentum range. To optimally stop the muons in our gas volume, we selected a beam momentum of $31.5 \mathrm{MeV} / \mathrm{c}$. Before entering the gas chamber, incoming muons were detected in a 1-mm-thick scintillator.

Fig. 2 shows a schematic muon view of the target and detectors. The gas chamber $\left(100 \times 115 \times 50 \mathrm{~mm}^{3}\right)$ is made of 15 -mm-thick aluminum covered with 5-7 $\mu \mathrm{m}$ gold. Four 10$\mu \mathrm{m}$-thick and 30-mm-diameter aluminized Mylar windows were installed to allow muons to enter the chamber and low energy X-rays to reach the two LAAPDs and the G1 germanium detector. Since LAAPDs are light sensitive, we chose aluminized Mylar in order to prevent the light to enter the chamber. The thickness was chosen such as to hold the slight overpressure in the chamber and to minimize the X-ray absorption. Apart from the LAAPDs, we used two germanium detectors to measure X-rays for monitoring purposes. G1 is a small volume $\left(0.17 \mathrm{~cm}^{3}\right)$ high purity germanium detector, suitable for X-ray detection in the 3-25 $\mathrm{keV}$ energy range and was essentially used to monitor if the muons were mainly stopping in the gas volume. G2 is a larger volume $\left(75 \mathrm{~cm}^{3}\right)$ high purity germanium detector, suitable for $70-1500$ $\mathrm{keV}$ energy range detection and was used to monitor the number of muons that interact in the 


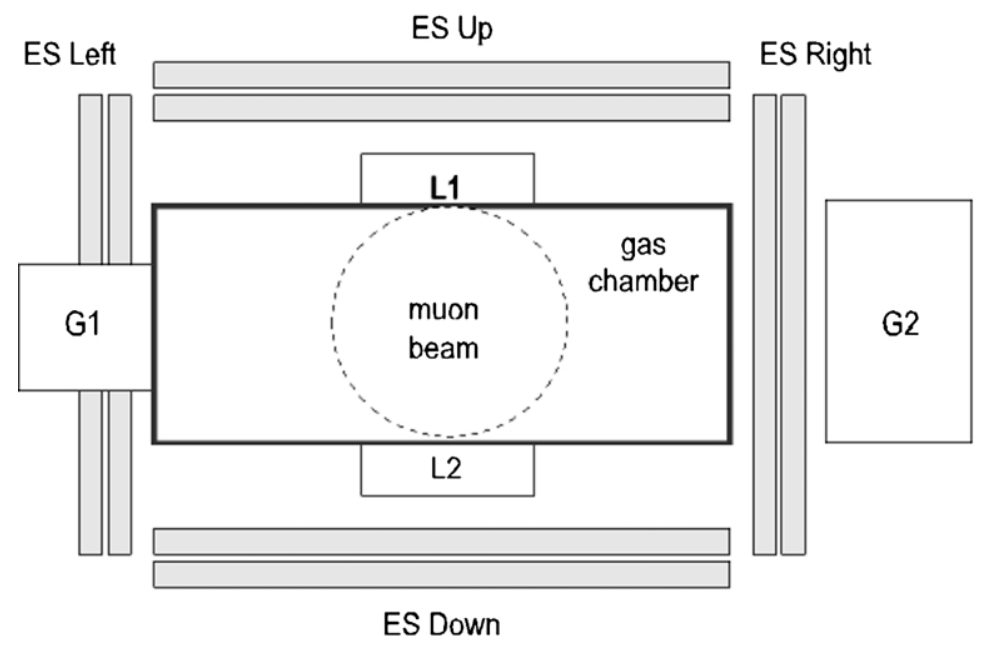

Fig. 2. Schematic muon view of the experimental system, showing the target and surrounding detectors.

chamber walls. This way, the muon beam momentum was optimized to maximize the muon stops in the gas and minimize the muon stops in the walls. No window was necessary in front of the G2 detector; however, the target wall thickness was reduced from 15 to $3 \mathrm{~mm}$.

Surrounding the target and the detectors, four pairs of scintillators (ES Left, ES Right, ES Up, ES Down) were installed to detect muon decay electrons. The scintillators have a planar shape and cover almost all space around the chamber, maximizing the solid angle for electron detection. Each scintillator (eight in total) has its own phototube. Using coincidence signals between two scintillator plates allows us to discriminate between heavy charged particles and electrons. Only the last ones can leave traces in both scintillator plates.

Different gases, such as hydrogen, helium and a mixture of hydrogen and methane, were used in our experiments. In all cases, a continuous gas flow was used.

LAAPDs, $16 \mathrm{~mm}$ in diameter, biased with 1800 $\mathrm{V}$, were used to detect the muonic X-rays produced in the gas. X-rays interact directly in the $\mathrm{Si}$ and the resulting primary electrons are amplified by the intense electric field around the junction, producing a pulse with amplitude that is, in average, proportional to the X-ray energy.
The LAAPD signals were fed through the Rutherford Appleton Laboratory (RAL) low-noise preamplifier [10], model $108-A$, to a spectroscopy amplifier and were pulse-height analyzed by a multi-channel analyzer. The $R A L$ 108-A preamplifier is a charge-sensitive thick ceramic board specially designed to amplify negative input signals and to give positive output signals. Short shaping time constants of $100 \mathrm{~ns}$ were used in the main amplifier to achieve an optimum energy resolution.

Only events taking place in coincidence with the signal given by the scintillator that monitors the muon entering the gas chamber (which set the time-zero) were recorded. For each LAAPD event, energy and time were recorded. Offline analysis permits any combination of energy and time from the different detector types to produce spectra.

\section{Results and discussion}

When muons are stopped in a gas volume, muonic atoms are produced in an excited state. Those atoms decay to the ground state and the subsequent X-rays can be detected. Muon collisions with the chamber walls produce $\gamma$ rays and electrons, contributing to the background. Additional delayed electrons are produced in consequence of the muon decay process. The muon 


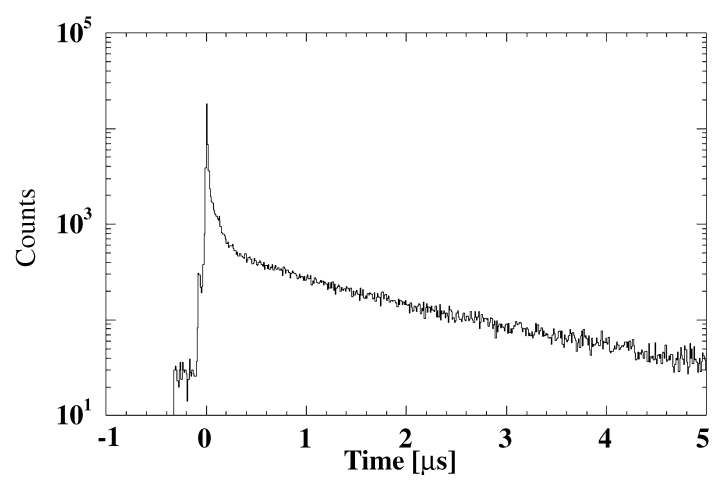

Fig. 3. Time spectrum for muonic hydrogen X-rays showing the exponential behavior of the muon decay process.

lifetime depends on the gas inside the chamber. The time spectra of LAAPD events show the exponential behavior characteristic of the muon decay, as shown in Fig. 3 for muonic hydrogen. An exponential fit would give the muon lifetime in the different elements of the target, namely, the gas and the walls. The muon lifetime in hydrogen is $2.2 \mu \mathrm{s}$ [11].

Since X-rays are produced just after the muon capture in a pure gas, we distinguish between prompt and delayed events, corresponding to events that take place at times shorter and longer than $30 \mathrm{~ns}$, respectively, in the attempt to reduce the X-ray background. The value $30 \mathrm{~ns}$ was chosen looking at energy and time spectra. Selecting only events in the useful energy range (between 1.5 and $2.5 \mathrm{keV}$ in muonic hydrogen) and checking the corresponding time, almost all events lie within \pm 30 ns. Fig. 4 presents different pulse-height distributions obtained with LAAPDs, for hydrogen and helium gases. The total distribution includes all events (X-rays and electrons) without any discrimination, while the prompt one includes only prompt events (time between \pm 30 ns relative to muon entrance). The resulting energy spectra from prompt events present a considerable background reduction, showing that the time discrimination is a powerful tool for effective X-ray background reduction. The background can be almost totally eliminated if a coincidence between the X-rays in the LAAPD and the corresponding electrons resulting from the muon decay, detected by scintillators within a 200-5200 ns time range, are further considered, as seen in the e-coincidence pulseheight distribution of Fig. $4 . \mathrm{K}_{\alpha}$ and $\mathrm{K}_{\beta} \mu \mathrm{He} \mathrm{X}$ rays can be efficiently discriminated from the electron background only when using coincidence with the muon decay electrons. However, the $\mathrm{X}$ ray counting rate drops by a factor of approximately 5 due to the discrimination parameters and the solid angle for the electron detection.

Fig. 5 depicts the total, prompt and electroncoincidence $\mathrm{X}$-ray pulse-height distributions for the $\mathrm{H}_{2}+4 \% \mathrm{CH}_{4}$ and $\mathrm{H}_{2}+16 \% \mathrm{CH}_{4}$ mixtures, obtained using the same discrimination timing parameters as in Fig. 4. For these mixtures, muons are captured either by hydrogen or carbon atoms.
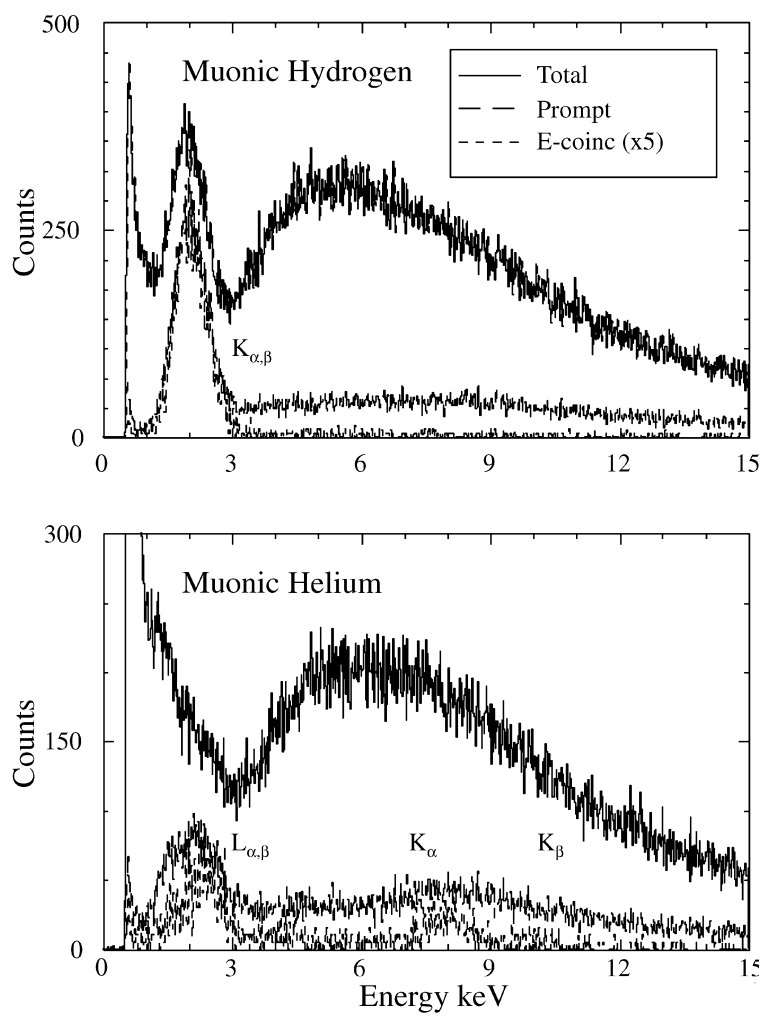

Fig. 4. Pulse-height distributions obtained with LAAPDs in two different pure gases. For hydrogen, the $1.89 \mathrm{keV} \mathrm{K}_{\alpha}$ and $2.25 \mathrm{keV} \mathrm{K}_{\beta} \mu \mathrm{p} \mathrm{X}$-rays are shown. For helium, the $8.23 \mathrm{keV}$ $\mathrm{K}_{\alpha}$ and $9.74 \mathrm{keV} \mathrm{K}_{\beta} \mu \mathrm{He} \mathrm{X}$-rays are visible, as well as the $1.52 \mathrm{keV} \mathrm{L}_{\alpha}$ and $2.05 \mathrm{keV} \mathrm{L} \mathrm{L}_{\beta} \mu \mathrm{He} \mathrm{X}$-rays. The big 'bump' above $3 \mathrm{keV}$ is due to electrons depositing energy in the LAAPD. 

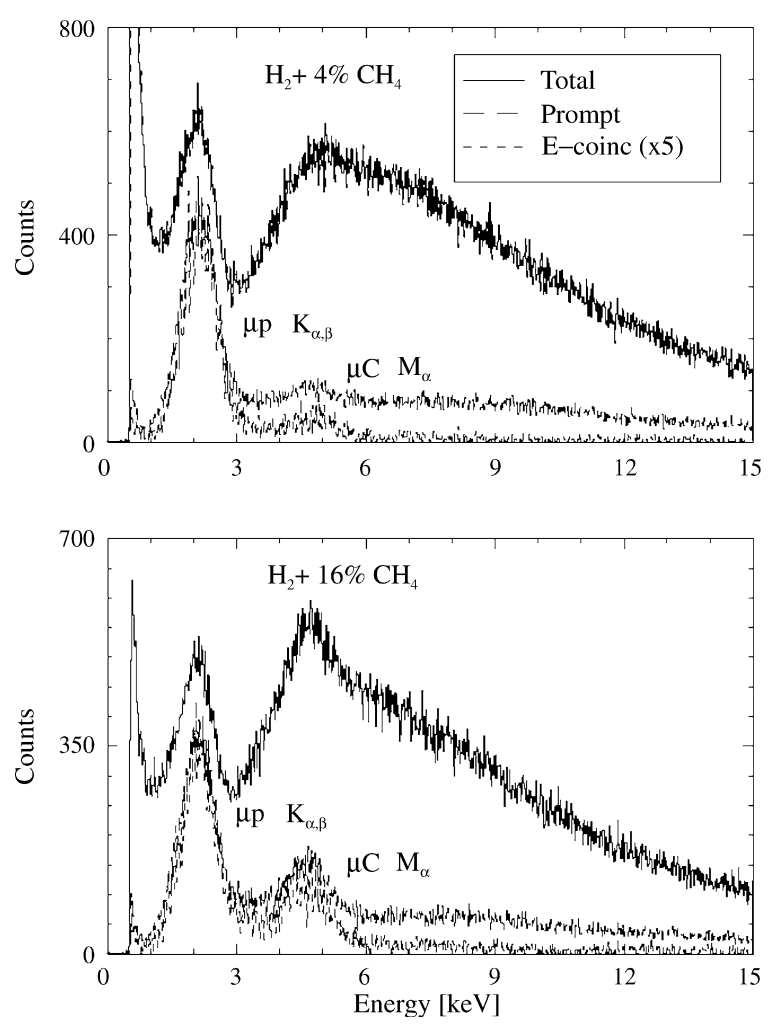

Fig. 5. Pulse-height distributions obtained for $\mathrm{H}_{2}+4 \% \mathrm{CH}_{4}$ and $\mathrm{H}_{2}+16 \% \mathrm{CH}_{4}$ mixtures. In both cases, $\mathrm{K}_{\alpha}$ and $\mathrm{K}_{\beta} \mu \mathrm{p}$ X-rays were detected, as well as the $4.88 \mathrm{keV} \mathrm{M}_{\alpha} \mu \mathrm{C}$ X-rays. As seen, $\mu \mathrm{C}$ peak intensities are directly dependent on the relative concentration of carbon in the mixture.

When hydrogen atoms capture muons, muon transfer to carbon takes place, with a reduced transfer rate of $0.95(5) \times 10^{11} / \mathrm{s}$ (relative to liquid hydrogen density) [12], producing delayed muonic carbon $(\mu \mathrm{C})$ atoms. Again, the e-coincidence spectra in Fig. 5 show the capability to identify the unique signature of muonic atom $\mathrm{X}$-rays that allow to effectively eliminate the background.

\section{Conclusions}

LAAPDs are alternative detectors for muonic atom X-ray spectrometry. Its compactness, insensitivity to intense magnetic fields and ionizing particles and radiation hardness are advantages to its use in the demanding environment of such experiments. However, its limited detection effi- ciency for X-rays with energies above $10 \mathrm{keV}$ may be a drawback in that case. Effective background reduction of the X-ray spectrum is achieved by discriminating the events taking place few tens of nanosecond after the muon entrance in the chamber. Additionally, the background can be effectively eliminated by further discriminating LAAPD events establishing its coincidence with the respective muon decay electrons.

\section{Acknowledgments}

Support is acknowledged to Fundação para a Ciência e a Tecnologia (FCT) through Project POCTI/FNU/41720/01 and to Swiss National Science Foundation. J.F.C.A. Veloso acknowledges support grant from FCT.

\section{References}

[1] D. Taqqu, F. Biraben, C.A.N. Conde, T.W. Haensch, F.J. Hartmann, P. Hauser, P. Indelicato, P. Knowles, F. Kottmann, F. Mulhauser, C. Petitjean, R. Pohl, R. Rabinowitz, R. Rosenfelder, J.M.F. Santos, W. Schott, L.M. Simons, J.F.C.A. Velosa, Laser spectroscopy of the Lamb Shift in muonic hydrogen, Hyp. Interact. 119 (1999) 311-315.

[2] F. Kottmann, W. Amir, F. Biraben, C.A.N. Conde, S. Dhawan, C. Donche-Gay, T.W. Haensch, F.J. Hartmann, V.W. Hughes, O. Huot, P. Indelicato, L. Julien, P. Knowles, S. Kazamias, Y.-W. Liu, F. Mulhauser, F. Nez, R. Pohl, P. Rabinowitz, J.M.F. dos Santos, L.A. Schaller, H. Schneuwly, W. Schott, D. Taqqu, J.F.C.A. Velosa, The muonic hydrogen Lamb Shift experiment at PSI, Hyp. Interact. 138 (2001) 55-60.

[3] P.J. Mohr, B.N. Taylor, Rev. Mod. Phys. 72 (2000) 351.

[4] S.G. Karshenboim, Can. J. Phys. 77 (1999) 241.

[5] L.M.P. Fernandes, J.A.M. Lopes, J.M.F. dos Santos, C.A.N. Conde, X-Ray Spectrom. 30 (2001) 164.

[6] M. Boucher, O. Huot, P.E. Knowles, L. Ludhova, F. Mulhauser, L.A. Schaller, C.A.N. Conde, J.M.F. dos Santos, L.M.P. Fernandes, J.F.C.A. Velosa, F. Kottmann, A. Antognini, R. Pohl, D. Taqqu, Large-area APDs for low energy X-ray detection in intense magnetic fields, Nucl. Instr. Meth. A. 505 (2003) 136-139.

[7] L.M.P. Fernandes, A. Antognini, M. Boucher, O. Huot, P.E. Knowles, F. Kottmann, L. Ludhova, F. Mulhauser, R. Pohl, J.M.F. dos Santos, D. Taqqu, J.F.C.A. Velosa, Behaviour of large-area avalanche photodiodes under intense magnetic field for VUV- visible and X-ray photon detection, Nucl. Instr. Meth. A. 498 (2003) 362-368. 
[8] L.M.P. Fernandes, J.A.M. Lopes, J.M.F. dos Santos C.A.N. Conde, 2003. X-ray spectrometry with Peltiercooled large-area avalanche photodiodes, Nucl. Instr. Meth. B, in press.

[9] Advanced Photonix Inc., 1240 Avenida Acaso, Camarillo, CA 93012, USA.

[10] CLRC Rutherford Appleton Laboratory, Chilton, Didcot, Oxfordshire, OX11, 0QX, England.
[11] T. Suzuki, D.F. Measday, J.P. Koalsvig, Phys. Rev. C 35 (1987) 2212.

[12] L. Schellenberg, A. Adamczak, R. Jacot-Guillarmod, F. Mulhauser, C. Piller, L.A. Schaller, H. Schneuwly, Y.A. Thalmann, S. Tresch, A. Werthmueller, Muon transfer to light atoms, Hyp. Interact. 101/102 (1996) 215-220. 